\title{
PREFACE
}

\section{A SPECIAL SELECTION ON BIOMECHANICAL APPLICATIONS IN MEDICAL SCIENCE - PART II}

\author{
Published 25 October 2021
}

This special selection on Biomechanical Applications in Medical Science presents the ongoing and active research in the Biomechanics area. The overall goal is to summarize recent discoveries and groundbreaking studies that will account for new challenge research in the broad and relevant area of Biomechanics used for medical science. The details of the accepted 24 papers are highlighted as follows.

Samwon Yoon et al. aim to examine the radiological difference in the affected knee joint and contralateral knee joint by analyzing unilateral older adults with medial knee osteoarthritis (OA) using both knees' joint space width (JSW) differences. This study shows no significant changes in the affected knee JSWs compared to the unaffected sides, but the difference between the lateral and medial JSWs is significant between the knees affected and unaffected by OA in the older adults with medial knee OA. These results show that the JSW is narrow, and the change between the lateral and medial sides increased in the radiographic images of knee OA.

Jiaqi Bi et al. investigate the influence of low-frequency electromagnetics (LFE) at different electromagnetic strengths on muscle cell recovery and assess the impact of chronic LFE on the immune system of Spinal cord injury (SCI) rats. High-energy LFE provoked intensive immune responses, while low-energy LFE did not affect immune responses. The mRNA and protein levels of Pax7 and MyoD are increased after LFE at both high and low electromagnetic strengths. The results demonstrate that LFE activates muscle satellite cells via stimulating myogenic factors. Chronic low-energy LFE is a safe therapy with no adverse impact on the immune system of SCI rats.

Chun-Chieh Liang et al. process the recent in-vivo survey results for over 1000 patients and optimized their neck and head computed tomography (CT) angiography triggered timing (CTA-TT) via the inverse problem algorithm, which ensures the maximal ratio of both left and right arterial to upper sinuses (LRA/US). These results are instrumental in examining the ischemic stroke syndromes along the neck and head. The CTA-TT can be successfully estimated by this formula without any 
preliminary test surveys of patients, which reduces their CT irradiation and radiologists' workload.

Fang Liu et al. built the model of lumbar disc and researched the nonlinear dynamic response of the system. A modified Van der Pol model is imported to describe the stress-strain curves of the lumbar disc. The system's dynamic model is set up, and the harmonic balance method is applied to revise the natural frequency of the system. The product of numerical simulation reveals that the lumbar disc has complex dynamic characteristics, including balance point, limit cycle bifurcation and stochastic Hopf bifurcation. By changing the parameters, we can avoid the largescale periodic movement of the lumbar disc which causes lumbar disc herniation $(\mathrm{LDH})$. These results contribute to the prevention of $\mathrm{LDH}$.

Jeong-Hyun Kim et al. made their research on the effectiveness of a 3D-modelingbased customized off-loading cushion to prevent pressure ulcers in people with SCI using wheelchairs. The CAD/CAM-based customized and traditionally manufactured customized off-loading cushions generated less average pressure on the IT and coccyx than the commercial adjustable air cushions. They conclude that the cushions manufactured using the $\mathrm{CAD} / \mathrm{CAM}$ and traditional methods exhibited no difference in clinical effects, but these cushions are more effective in preventing pressure ulcers than the adjustable air cushion.

Tengda Zhou et al. propose a one-dimensional (1D) U-net++ to identify the position of $J$-peak in ballistocardiogram (BCG) signals automatically. The proposed $1 \mathrm{D}$ U-net++ is based on 1D convolution neural network through dense skip connection backward transfer data features. The BCG signals of eight healthy subjects are collected for experimental verification, and the accuracy and precision of $J$-peak detection reach $99.4 \%$ and $99.3 \%$, respectively. The experimental results demonstrate that our proposed method can effectively identify the $J$-peak in the BCG signal. In the future, simultaneous electrocardiogram (ECG) acquisition with recorded BCG signal will be addressed for further clinical research.

Junyi Yan et al. subdivide the human brain into several functionally distinct and spatially contiguous areas which are important to understand the amazingly complex human cerebral cortex and they fuse these population average matrixes to obtain the multi-modal similarity matrix and feed it into the spectral clustering algorithm to obtain the brain parcellation for each age group. The parcellation result shows most of the areas showing consistency across different age groups, such as the somatosensory area and the visual area. We hope the derived functional parcellation could promote the understanding of the development of human brain aging.

Xiaokun Li et al. systematically evaluate the effectiveness of Baduanjin in the treatment of LDH by collecting the randomized trials (RCTs) of Baduanjin in the treatment of $\mathrm{LDH}$. After literature screening, data extraction and quality evaluation, the RevMan 5.4 software is used for meta-analysis and 13 studies involving 853 patients are finally included in the research. The results show that compared with the control group, Baduanjin can significantly improve the rehabilitation evaluation 
scores of LDH patients and the treatment efficiency, and its curative effect is more obvious in a long time.

Maria Kong et al. investigate the effects of functional games using neurofeedback on cognitive function and changes in brain waves of people with developmental disabilities. The neurofeedback training program applied in this study is a noninvasive method of developing the brain using brain waves. This study provides empirical evidence that functional games using neurofeedback are effective in improving the cognitive function of adults with developmental disabilities. In addition, this improvement of cognitive function is meaningful in that it can exert a positive effect on learning communication skills and improving quality of life.

Lei Wang et al. detect the changes in brain microstructure in patients with Parkinson's disease (PD) using diffusion kurtosis imaging (DKI) to quantitatively diagnose early-stage PD. Because the MK values in the substantia nigra correlate with the severity of motor dysfunction in patients with $\mathrm{PD}$, regular MK detection may help in evaluating the progression of PD. As a sensitive index, the MK in the substantia nigra is a potential biomarker for imaging studies of PD. However, future studies also need to research MK in the basal ganglia.

Hong Zhang et al. propose that motor activations evoked by emotional processing influence the activations in limbic areas such as amygdala during the perception of facial expressions. A masked priming paradigm is adopted in our fMRI experiment, which could modulate the activation within motor cortex when healthy participants perceive sad or happy facial expressions. This work reveals the modulation effect of motor cortex on brain regions related to emotion perception. Our results provide new evidence for the function role of motor system in perception of facial emotions and could contribute to the understanding of the deficit in social interaction for patients with autism or schizophrenia.

Zeguo Shao et al. formulate the strategies for emotional nursing, sports rehabilitation nursing and interventions for poor lifestyle habits such as smoking, drinking and picky eating for patients with stroke at home. Data are obtained through tracking investigation, effect evaluation indexes are developed according to Hamilton depression scale (HAMD), activities of daily living (ADL) and other rating scales; C4.5 decision tree algorithm is used to analyze the effect of nursing intervention strategy. It can be seen that it's an effective way to promote rehabilitation to relieve the anxiety and depression of patients through emotional nursing and intervention.

Chanhee Park et al. demonstrate the validity, feasibility and reliability of the progressive exoskeletal robotic shoulder joint kinematics system. The changes in the shoulder joint angle between ImageJ motion analysis software and robotic shoulder joint kinematics system are compared in this study to investigate the validity and reliability of the latter. This study shows that the RSJK system is reliable and accurate for measuring the shoulder joint angular movements. The proven validity and test-retest reliability of the novel RSJK system will empower the precise treatment of kinematic movement disorders in patients with hemiparetic stroke and $\mathrm{CP}$, experiencing abnormal flexor synergy and spasticity. 
Chao-Yu Chiang et al. evaluate the minimum detectable difference (MDD) at various beats/min (BPMs) of CT angiography (CTA) using an oblique V-shape line gauge and PMMA phantom in this study. The reciprocating mechanism in the phantom is run by a step motor with an eccentric gear connected to a crank rod to provide a stable harmonic motion, simulating the cardiac beats. The MDD proves to be a useful and practical technique for justifying the CTA scanned images.

Zhenghao Shi et al. present an arrhythmia classification with ECG based on deep residual, of which two improved residual blocks are used to combine soft and hard subsampling. With such blocks, the network can well hold spatial information and improve the classification performance with a simple model structure. Experiments on the MIT-BIH arrhythmia database show that the proposed method obtains an average classification accuracy of $99.59 \%$ and an average classification specificity $99.63 \%$, which are $0.26 \%$ and $0.57 \%$ higher than that of the most state-of-the-art method based on deep learning. In the future, it is necessary to consider more arrhythmias in clinical practice of the department of cardiology.

Mi Li et al. put forward a method for depression recognition using resting-state functional magnetic resonance imaging (rest_fMRI). The rest_ReHo_fMRI is extracted from the preprocessed rest-fMRI by calculation. Deep convolutional networks (such as VGG16) pretrained on ImageNet are used to automatically complete extracting the classification features from rest_ReHo_fMRI. The kernal extreme learning machine (KELM) is used to classify depression. The results of the test set show that the proposed method achieves $89.07 \%$ in sensitivity and $89.74 \%$ in specificity. This study suggests that the features of rest_ReHo_fMRI can be used as biomarkers to distinguish depression from normal people.

Peng An et al. analyze the anatomical morphological and hemodynamic characteristics of left ventricular outflow tract stenosis (LVOTS) by echocardiography and magnetic resonance imaging (MRI). MRI enhancement can be used for the quantitative measurement of myocardial fibrosis. Echocardiography can distinguish the stenosis types of LVOTS. It can help cardiologists quickly determine the cause, type and degree of LVOTS by using two-dimensional color Echocardiography and M-mode Doppler to detect the spectrum and ejection peak values, combined with MRI to measure the LV motion indexes and quantitative assess myocardial fibrosis.

Changle Li et al. intend to establish a robotic system in puncture surgery, which is based on optical registration to improve safety, accuracy and efficiency. As the accuracy of surgical space calibration influences the accuracy of the surgical system, this study proposes an improved automatic calibration algorithm for linear rotation. The algorithm can reduce the error caused by manual calibration and system noise. Recalibration is not required provided that the pose of the digital reference frame is unchanged, thereby improving accuracy and efficiency. Results show that the average errors of position and posture are $0.25 \mathrm{~mm}$ and $0.2^{\circ}$, respectively. The accuracy of calibration fully meets the needs of surgery.

Jingtong $\mathrm{Yu}$ et al. studied the protein expression levels and morphological structure in the hippocampi of mice after exposure to SEF so as to explore the effects 
of static electric field (SEF) generated by ultra-high-voltage direct-current (UHVDC) transmission lines. After the exposure, hippocampal Ca2+/calmodulindependent protein kinase II (CaMKII) and calcineurin (CaN) expression levels are assayed by Western blot. The results show that the hippocampal neuron ultrastructure damage may be a mechanism of SEF-exposure-induced memory decline in mice.

Wansong $\mathrm{Xu}$ et al. propose a two-lead joint detection algorithm of QRS complexes to improve the detection ability of QRS complexes under sudden interference and study the QRS complex information on multiple leads in depth. The proposed algorithm has high accuracy, can effectively deal with the sudden interference of ECG signal. The two-lead joint detection algorithm can be used in standard clinical ECG and household portable ECG monitor to fully mine the QRS complexes information in multiple leads, so as to improve the ability of anti-signal mutation and improve the correct detection rate.

Mi Yeon Shin et al. analyze the hydrogen emission characteristics of a hydrogen storage alloy operating system employing the Zr0.9Ti0.1Cr0.6Fe1.4 alloy under different temperature and loading conditions for application in a rehabilitation system for motion assistance for the aged and disabled. The hydrogen storage alloy operating system developed in this study is expected to be useful in motion assistance for the socially underprivileged, as a rehabilitation tool for the hand, wrist, and elbow, as supplementary equipment, and in rehabilitation systems for daily life.

Mingliang Liu et al. demonstrate a new type of shock-absorbing structure that can be used to improve the stability of the ventilator's gas pipeline, thereby reducing the risk of the gas pipeline falling due to external interference. The numerical and experimental results show that the position of SMA can change the system's stability, the proposed structure can reduce the system's vibration to the maximum extent. The new composite structure proposed in this paper has a positive significance for the stability of the ventilator and brings more help to severe patients with new coronavirus pneumonia.

Chun-Chieh Wang et al. assess the absorbed dose for patients who undergone Tc$99 \mathrm{~m}$ methylene diphosphonates (MDP) bone scan examination based on a series of personal dosimeter measurements and a derived semi-empirical formula. Five biological parameters (gender, age, BMI, eGFR and creatinine) are collected and processed by the STATISTICA program, yielding a nonlinear 16-term first-order semi-empirical formula of the residence time. This methodology can be recommended for other hospitals for public hygiene and safety enhancement.

Yeongsang An et al. develop an innovative deep learning-based smartphone application Funrehab Exercise Game (FEG) to provide accurate kinematics movement and motivation as well as high-intensity and repetitive movements using deep learning. They compared the effects of continuous passive movement (CPM) and FEG on brain activity and shoulder range of motion in patients with adhesive capsulitis (AC). The outcome measures are shoulder abduction kinematics movement and electroencephalography (EEG) brain activity during the intervention. The 
results provided a novel and promising clinical evidence that FEG can more effectively improve neurophysiological EEG data and shoulder abduction movements than CPM in patients with AC.

To sum-up, this special issue is a continuous effort in promoting the biomechanical application in medical diagnosis and treatment, ${ }^{1-8}$ including rehabilitation engineering, finite element modeling and biofluid mechanics etc. in conjunction with Biomedical Imaging. ${ }^{9-21}$ As guest editors, we sincerely hope that the selected papers will be of long-lasting value to biomechanics, and we believe that it could allow some insights for the challenging field of Biomechanical Applications in Medical Science and relevant areas.

Guest Editors

Esteban Peña Pitarch

Universitat Politècnica de Catalunya (UPC), Spain

Eddie Y. K. Ng

Nanyang Technological University, Singapore

\section{References}

1. Liu F, Ng EYK, Chen Z, A special section on biological mechanics, J Mech Med Biol 15(6):1502002-1, 2015.

2. Liu F, Ng EYK, A special section on biological mechanics, J Mech Med Biol 16(8):1602002-1, 2016.

3. Liu F, Ng EYK, A special section on biological mechanics, J Mech Med Biol 17(7):1702002-1, 2017.

4. Peña E, Drochon A, Ng EYK, A special selection on biological applications of mechanics, J Mech Med Biol 18(7):1802001-1, 2018.

5. Peña E, Drochon A, Ng EYK, A special selection on biological applications of mechanics, J Mech Med Biol 18(8):1802002-1, 2018.

6. Peña E, Drochon A, Ng EYK, A special selection on biomechanics in medical application (Part 1), J Mech Med Biol 19(7):1902003-1, 2019.

7. Peña E, Drochon A, Ng EYK, A special selection on biomechanics in medical applications (Part 2), J Mech Med Biol 19(8):1902004, 2019.

8. Peña E, Drochon A, Ng EYK, A special selection on mechanical engineering applied to biomedicine (Part 1), J Mech Med Biol 20(9):2002001, 2020.

9. Peña E, Drochon A, Ng EYK, A special selection on mechanical engineering applied to biomedicine (Part 2), J Mech Med Biol 20(10):2002002, 2020.

10. Liu F, Ng EYK, A special section on biomedical imaging in diagnosis and treatment (Part 1), J Med Imag Health Inf 6(5):1209-1211, 2016.

11. Liu F, Ng EYK, A special section on biomedical imaging in diagnosis and treatment (Part 2), J Med Imag Health Inf 16(7):1670-1672, 2016.

12. Liu F, Ng EYK, A special section on biomedical imaging in diagnosis and treatment (Part 3), J Med Imag Health Inf 17(1):126-128, 2017.

13. Liu F, Ng EYK, A special section on methods and application in biomedical imaging (Part 1), J Med Imag Health Inf 7(5):919-921, 2017.

14. Liu F, Ng EYK, A special section on methods and application in biomedical imaging (Part 2), J Med Imag Health Inf 7(7):1522-1524, 2017. 
15. Liu F, Ng EYK, A special section on methods and application in biomedical imaging (Part 3), J Med Imag Health Inf 8(1):1-4, 2018.

16. Gomez L, Ng EYK, A special section on methods and application in biomedical imaging (Part 1), J Med Imag Health Inf 8(7):1364-1367, 2018.

17. Gomez L, Ng EYK, A special section on methods and application in biomedical imaging (Part 2), J Med Imag Health Inf 8(8):1607-1610, 2018.

18. Gomez L, Ng EYK, A special section on methods and application in biomedical imaging (Part 3), J Med Imag Health Inf 9(1):43-46, 2019.

19. Gomez L, Ng EYK, A special section on methods and application in biomedical imaging (Part 1), J Med Imag Health Inf 9(7):1415-1417, 2019.

20. Gomez L, Ng EYK, A special section on methods and application in biomedical imaging (Part 2), J Med Imag Health Inf 9(9):1849-1852, 2019.

21. Gomez L, Ng EYK, A special section on emerging techniques for biomedical imaging (Part 3), J Med Imag Health Inf 10(3):610-613, 2020.

22. Gomez L, Ng EYK, A special section on emerging techniques for biomedical imaging (Part 1), J Med Imag Health Inf 10(11):2660-2662, 2020. 